\title{
Estudio neuropsicológico del sistema de memoria en pacientes adultos de edad intermedia con trastorno mixto ansiedad- depresión
}

\author{
Study neuropsycologic memory system in a sample of middle \\ age adults with mixed anxiety-depression disorder
}

\author{
César Sarria Joya ${ }^{1 *}$, Victoria Llaja Rojas², Víctor Montero López ${ }^{3}$, \\ Pedro García Pizarro ${ }^{4}$, Marina Salazar Cahuana ${ }^{5}$ \\ Universidad Nacional Mayor de San Marcos
}

Recibido: $13-09$ - 16

Aceptado: $01-06-17$

\section{Resumen}

El objetivo de la investigación es describir y analizar los trastornos del Sistema de Memoria de pacientes diagnosticados con depresión y/o ansiedad, haciendo un estudio comparativo con un grupo control de personas sin disfunción en esta esfera afectivo-emotiva; y explicar el compromiso de su actividad funcional mediante el modelo teórico informacional (Ortiz, 1994,1995,1997, 1997b, 1998, 1999, 2002). La muestra estuvo conformada por todos los pacientes diagnósticados por el Neuropsicólogo Clínico , los cuales han sido derivados para evaluación y tratamiento, al Servicio de Neuropsicología del Hospital Edgardo Rebagliati ,y el grupo control estuvo configurado por sujetos que no presenten dicha alteración, y que sean homogéneos en los datos demográficos con la muestra clínica. Se utilizó el Test de Recuerdo Selectivo de Herman Busckhe y Paula Altman, adaptado y validado en el medio por Llaja $(1998,2000)$ en una muestra de pacientes con Amnesia Global Transitoria, y en niños de 5 años respectivamente. Se recogieron los datos durante el periodo de enero a noviembre de 2011. El diseño fue cuasiexperimental con 2 grupos según clasificación de Hernandez, Fernandez y Baptista (2010). Se administró además la Escala de Zung de Ansiedad/Depresión para determinar el grado de disfunción en la muestra estudiada. Asi mismo se interpretó los hallazgos a través del Modelo TIP, que viene demostrando su validez en el estudio clínico neuropsicológico integral de estos desordenes.

Palabras clave: Sistema de memoria,test de recuerdo selectivo, desorden mnésico, sistema de personalidad, modelo TIP.

\footnotetext{
*Autor para correspondencia

Facultad de Psicología, Universidad Nacional Mayor de San Marcos.

1. Email: csarriaj@unmsm.edu.pe

2. Email: vllajad@unmsm.edu.pe

3. Email: vmonterol@unmsm.edu.pe

4. Email: pgarciap@unmsm.edu.pe

5. Email: msalazarc@unmsm.edu.pe
} 


\begin{abstract}
The objective of this research is to describe and analyze the memory system disorders of patients diagnosed with depression and/or anxiety, making a comparative study with a control group of people without dysfunction in this area affective-emotional ; and explain the commitment of its functional activity using the theoretical model informational (Ortiz, 1994,1995,1997, 1997b, 1998, 1999,2002). The sample consisted of all patients diagnosed by the clinical neuropsychologist, which have been referred for evaluation and treatment, in the Service of Neuropsychology of Hospital Edgardo Rebagliati and the control group was set up by subjects who do not submit such alteration, and that they are homogeneous in the demographic data with the clinical sample. We used the Busckhe Selective Memory of Herman and Paula Altman, adapted and validated in the middle by 1998 Llaja, V., 2000 in a sample of patients with transient global amnesia, and in children of 5 years respectively. Data were collected during the period January to Nov 2011-. The design was quasi-experimental design, with 2 groups according to the classification of Hernández, Fernández \& Baptista (2010). Was administered in addition to the Zung Scale of Anxiety/Depression to determine the degree of dysfunction in the studied sample. It is also interpreted the findings through the Model TIP, which is demonstrating their validity in the comprehensive neuropsychological clinical study of these disorders.
\end{abstract}

Keywords: Memory system, selective memory disorder, personality system, TIP model.

El Síndrome Amnésico ha sido ampliamente estudiado a nivel internacional en Neuropsicología clínica desde la perspectiva luriana (Luria, 1974) que enunciaba que el estudio de los procesos de retención y su alteración en los casos de estados patológicos del cerebro pueden arrojar valiosos resultados para el estudio clínico de las lesiones focales del cerebro. Según Keefover. (1998, citado en Luria, 1980) los cambios en la memoria que tienen lugar a través del tiempo se manifiestan en : recuerdo diferido(retención por minutos a horas) recuerdo libre (ítems que se recuerdan sin la ayuda de "cues" o estímulos asociados), memoria episódica (recuerdo de información en un contexto específico), memoria explícita (recuerdo consciente de información específica), y memoria de trabajo (retención de información para manipulación). .Asimismo, de acuerdo al Psicólogo Miller (1958, citado en Luria, 1980) la amplitud de la memoria mide la cantidad de un material dado que puede ser reproducido después de una sola lectura. Este autor ha formulado la hipótesis de que el hombre promedio puede procesar alrededor de 7 bits de información a la vez y esta capacidad limitada de procesamiento de la memoria nos obliga a abreviar y condensar la información a través de un proceso llamado troceado o "chunking", al hacer trozos usamos una especie de taquigrafía mental para codificar y almacenar los ítems. Los niveles de organización a que llegue el proceso de recordación dependerán de la tarea que el sujeto tiene ante sí, del carácter del material a recordar, y del tiempo que se dé al sujeto para su registro.

Aquí podemos citar algunos estudios efectuados con el instrumento seleccionado para examinar a nuestros pacientes en la presente investigación, realizados por Llaja $(1983,2000)$ en los que se obtuvo conclusiones respecto al Sindrome de Amnesia Global Transitoria estableciendo un diagnóstico diferencial con otras entidades neurológicas. Uno de los hallazgos más relevantes en este estudio piloto fue que a través del Test de Recuerdo Selectivo se pudo diferenciar 
el desempeño mnémico de pacientes con amnesia global transitoria cuya etiología fue psicógena, y aquellos que tenían patología estructural, tipo isquemia cerebral transitoria. Lo característico de este síndrome es que no deja secuela, a excepción del episodio de amnesia, y algunos pacientes presentaron más de un episodio recurrente de amnesia. De otro lado se trabajó con este mismo instrumento en niños de cinco años donde se relacionaron los estímulos lingüísticos familiares: elaborados y restringidos y la memoria verbal en donde una de las conclusiones más relevantes es que se encontró diferencias significativas entre los niños en cuanto a su desempeño de memoria verbal, específicamente en las categorías de memoria a corto plazo y largo plazo, y el desempeño favorecía a los niños que eran estimulados con códigos elaborados.

En nuestra realidad estos estudios son infrecuentes, y el presente constituye un aporte más en el campo de la neurociencia.

\section{JUSTIFICACIÓN}

Este estudio se justifica en la medida que la labor de prevención y promoción de la salud dentro del campo de la neuropsicología clínica, es una de las tareas de vital trascendencia en estos tiempos debido a que las enfermedades psicosomáticas y otras de causa psicógena han tenido un incremento notable en la última década, así el estrés ha sido considerado como el flagelo de este siglo y hay mayor incidencia actualmente de estos trastornos en la población adulta intermedia. Conociendo las características del síndrome amnésico y la alta incidencia asociada a disfunciones en los procesos afectivo-emotivos, sea que se trate de trastornos funcionales o estructurales como consecuencia de una patología cerebral, estaremos contribuyendo a reducir su prevalencia y podemos recomendar medidas terapéuticas y de restitución más óptimas para el abordaje integral de esta casuística.

El objetivo general de la investigación es describir las características del Síndrome Amnésico asociado al trastorno mixto ansiedad-depresión, y explicar los hallazgos bajo el Modelo TIP. De acuerdo con ello, nuestro objetivos específicos son:

1. Estimar cual de las cuatro fases del proceso de memorización se encuentra más comprometida en la muestra clínica estudiada.

2. Determinar si existen diferencias significativas en el proceso de memorización de nuestros pacientes y del grupo control sin disfunción de los procesos afectivo-emotivos.

3. Corroborar si la evaluación neuropsicológica integral del sistema de memoria a través del Test de Recuerdo Selectivo sigue siendo una medida diagnóstica válida y confiable de los desordenes del Sistema de Memoria asociados a trastornos de naturaleza psicógena. 
Esto servirá para: a) realizar una medida diagnóstica eficiente y válida sobre los desordenes del sistema de memoria asociados a trastornos de naturaleza psicógena; b) determinar si existen diferencias en las fases del proceso de memorización de nuestra muestra clínica y el grupo control que puedan ser detectadas a través del Test de Recuerdo Selectivo a fin de intervenir oportunamente, con programas de prevención y promoción de la salud en la adultez intermedia; c) contar con un instrumento que sea sensible para la detección de los déficits de memorización asociados a trastornos de naturaleza psicógena, y que pueda establecer diagnóstico diferencial con aquellos de patología cerebral, a fin de realizar estrategias de diagnóstico e intervención oportuna en los déficits del sistema de memoria que en el momento actual tienen alta incidencia en el medio.

Con este estudio pretendemos contribuir al diagnóstico preciso de desordenes neuropsicológicos del sistema de memoria asociados a trastornos afectivoemotivos de naturaleza psicógena, frecuentes en este siglo en la población adulta intermedia. asimismo difundir los hallazgos encontrados en la muestra clínica mediante el Test de Recuerdo Selectivo, instrumento idóneo y completo de la Bateria Neuropsicológica de EsSalud (Unidad de Neuropsicología), para establecer los déficits de todas las fases del proceso de memorización, ya que ha sido validado en estudios anteriores en otras casuísticas clínicas y educativas, pero este estudio es original e inédito ; en una muestra de pacientes con trastorno mixto ansiedad-depresión. Así mismo se podrá precisar el diagnóstico diferencial con otras entidades neuropsicológicas de base estructural, de allí que consideramos que es un aporte de impacto en la neuropsicología; y es el punto de partida de otras investigaciones que generarán medidas de prevención y promoción de la salud en esta población.

Nuestra hipótesis general es que los pacientes con trastorno mixto ansiedaddepresión presentan desorden en el sistema de memoria, estudiados a través del Test de Recuerdo Selectivo; mientras que las hipótesis específicas son las siguientes:

1. Los pacientes con trastorno mixto ansiedad-depresión, presentan déficit en los procesos de registro a corto plazo.

2. Los pacientes con trastornos mixto-ansiedad-depresión, presentan déficit en los procesos de almacenamiento a largo plazo..

3. Los pacientes con trastornos mixto ansiedad-depresión, presentan déficit en la consolidadación y evocación de datos a largo plazo.

Finalmente, ambicionamos llamar la atención de otros investigadores del campo de la neuropsicología clínica, tratando de generar efecto multiplicador en el abordaje de otros estudios similares que puedan cambiar la realidad en esta línea de trabajo con rostro humano. 


\section{MÉTODO}

La investigación es de tipo exploratorio, según la clasificación de Díaz, (2009). Las hipótesis son de tipo descriptivo. Se empleará un diseño cuasiexperimental: con preprueba-posprueba y grupos intactos (uno de ellos de control) según la clasificación de Hernandez, Fernandez y Baptista (2010). Para la recolección de la muestra se utilizò un muestreo no probabilistico intencional. Como criterio de inclusión se evaluò pacientes con trastorno mixto ansiedad-depresión, fueròn excluidos todos aquellos que posean otra patología psicógena y/ o estructural. Se reclutaron todos los pacientes nuevos que fueron derivados a la Consulta Externa del Servicio de Neuropsicología por los médicos especialistas (de Neurología o Salud Mental).

\section{PROCEDIMIENTO}

Se realizaròn las coordinaciones previas con los médicos especialistas para que sean derivados los pacientes a la evaluación Neuropsicológica.

Se elaborò la anamnesis clínica previa, luego se administrò la Escala de Depresión/ansiedad de Zung a fin de corroborar el trastorno mixto Ansiedad Depresión, y seguidamente el Test de Recuerdo Selectivo de Buschke \& Altman, para examinar el desempeño mnésico, de los 2 grupos en tres sesiones en un ambiente excento de estímulos distractores. Se corroboraron algunos datos con testigos o familiares directos, para tener una aproximación objetiva a su línea basal de la actividad mnésica y a la corroboración de signos y sintomas de los procesos afectivo-emotivos.

Se aplicò el paquete estadístico SPSS Versión 17 para el análisis de datos, con estadísticos descriptivos básicos (media y desviación estándar, la $t$ de student para la comparación de los hallazgos en el grupo clínico y el grupo sin trastorno de las esferas afectivo.emotivas. Por tratarse del sistema de memoria, y siendo importante tomar en cuenta la temporalidad del disturbio, se hizo una sistematización parcial en cuanto a la equivalencia de los datos demográficos de ambos grupos. .

Asimismo se realizo la interpretación cualitativa, de corte neuropsicológico bajo el modelo TIP, de los hallazgos neuropsicológicos para contestar a las hipótesis propuestas, y arribar a las conclusiones con el rigor metodológico suficiente para que este estudio tenga validez tanto interna como recursos de generalización externa.

\section{RESULTADOS}

A continuación se describen las Tablas 1 y 2 de la configuración de la muestra.

En la tabla 1 observamos la distribución de la muestra de acuerdo a su atención o no en el Servicio de Neuropsicología del Hospital Edgardo Rebagliati M., encontrando que la mayoría pertenece a población no atendida en dicho 
establecimiento, representado por un 50.3\%.La muestra total estuvo constituida por 165 participantes

\section{Tabla 1}

Descripción de la muestra de acuerdo a si son atendidos o no en el servicio de neuropsicología del Hospital Edgardo Rebagliati.

\begin{tabular}{|c|c|c|}
\hline & $\mathbf{F}$ & $\%$ \\
\hline Clínico & 82 & 49.7 \\
\hline No clínico & 83 & 50.3 \\
\hline Total & 165 & 100.0 \\
\hline
\end{tabular}

Asimismo aquí observamos la distribución de la muestra de acuerdo al género, siendo la mayoría mujeres, representado por un $57,6 \%$ y los varones con un $42,4 \%$.

Tabla 2

Descripción de la muestra según género

\begin{tabular}{lll}
\hline & F & \% \\
\hline Varón & 70 & 42.4 \\
Mujer & 95 & 57.6 \\
Total & 165 & 100.0 \\
\hline
\end{tabular}

En la tabla 3 no se observa relación estadísticamente significativa entre la mayoría de categorías de memoria y la presencia de la ansiedad, a excepción del área RCP, que presenta una correlación altamente significativa con una significancia $<0.05$, en los pacientes del servicio de neuropsicología del Hospital Edgardo Rebagliati. Martins.

\section{Tabla 3}

Relación entre los tipos de memoria y la ansiedad.

\begin{tabular}{cccccccc}
\hline Ansiedad & SR & ALP & RLP & APC & RLPA & RCP & NP \\
\hline $\mathrm{r}$ & -.215 & -.188 & -.172 & -.172 & .085 & $.333(* *)$ & .144 \\
Sig. & .081 & .127 & .165 & .163 & .492 & .006 & .246 \\
\hline
\end{tabular}

En la tabla 4 no se observa relación estadísticamente significativa entre la mayoría de categorías de memoria y la presencia de depresión, a excepción del área RCP, que presenta una correlación altamente significativa con un $\mathrm{p}<0.05$, en los pacientes del servicio de neuropsicología del Hospital Edgardo Rebagliati Martins. 
Tabla 4

Relación entre los tipos de memoria y la depresión.

\begin{tabular}{cccccccc}
\hline Zung Dep. & SR & ALP & RLP & APC & RLPA & RCP & NP \\
\hline r de Pearson & -.131 & -.105 & -.062 & -.085 & .144 & $.264\left(^{*}\right)$ & .210 \\
Sig. & .290 & .399 & .621 & .494 & .245 & .032 & .088 \\
\hline
\end{tabular}

En la tabla 5 observamos que existen diferencias significativas entre los que se atienden en el servicio de neuropsicología del Hospital Edgardo Rebagliati y los que no, en las diferentes categorías del sistema de memoria, favoreciendo a los no clínicos.

Tabla 5

Comparación de las categorías del sistema de memoria desarrollado en pacientes y no pacientes del Servicio de Neuropsicología del Hospital Edgardo Rebagliati.

\begin{tabular}{lllll}
\hline & sujetos & N & Media & Sig. \\
\hline SR & clínico & 81 & 6.23 & 0.00 \\
& no clínico & 83 & 7.47 & \\
ALP & clínico & 81 & 6.16 & 0.00 \\
& no clínico & 83 & 7.86 & \\
RLP & clínico & 81 & 5.70 & 0.00 \\
& no clínico & 83 & 6.98 & \\
APC & clínico & 81 & 3.14 & 0.00 \\
& no clínico & 83 & 5.78 & \\
RLPA & clínico & 81 & 2.94 & 0.00 \\
& no clínico & 83 & 1.47 & \\
RCP & clínico & 80 & 2.11 & 0.00 \\
& no clínico & 83 & .46 & \\
NP & clínico & 81 & 4.77 & 0.00 \\
& no clínico & 83 & 3.47 & \\
\hline
\end{tabular}

\section{DISCUSIÓN}

En el presente estudio se ha registrado que en la muestra de pacientes con Diagnóstico de trastorno mixto ansiedad-depresión (82 pacientes) no existe relación significativa entre la disfunción de los procesos afectivo-emotivos (ansiedad-depresión) y su desempeño mnésico, lo que indica que los pacientes se desenvuelven adecuadamente en este Test, aún cuando muestran dicho estado emocional. Su rendimiento no se ve afectado en ambos casos, a excepción del recuerdo a largo plazo, el cual requiere de niveles de atención mucho más exigentes; esto corrobora, que los olvidos que dicen manifestar nuestra muestra de estudio puede ser atribuido a un déficit de atención más que a un trastorno primario de la memoria.

Estos hallazgos han sido objetivados análogamente en la investigación realizada por Llaja (1983) en la que la muestra clínica de pacientes con amnesia 
global transitoria no se diferenciaba en su desempeño, con la muestra de sujetos con Diagnóstico de naturaleza psicógena, porque en los primeros no quedaba secuela en el sistema de memoria.

Así mismo como afirman algunos autores que la atención es un proceso, y señalan que presenta fases entre las que podemos destacar la fase de orientación, selección y sostenimiento de la misma. (Ardila, 1979; Celada, 1989; Cerdá, 1982; Luria, 1986; Taylor, 1991; mencionados en Sarria, García y Llaja, 2008, p.76)).

Reategui (1999 mencionado en Sarria, García y Llaja op.cit., p. 52) señala que la atención es un proceso discriminativo y complejo que acompaña todo el procesamiento cognitivo, además es el responsable de filtrar información e ir asignando los recursos para permitir la adaptación interna del organismo en relación a las demandas externas.

Otros consideran que la atención es un mecanismo, va a poner en marcha a los procesos que intervienen en el procesamiento de la información, participa y facilita el trabajo de todos los procesos cognitivos, regulando y ejerciendo un control sobre ellos (García, 1997; Rosselló, 1998; Ruiz-Vargas, 1987; mencionados en Sarria, García y Llaja, 2008, p.74).

Para Celada (1990; mencionado en Ortiz, 1994 p. 54) el cambio de la atención es intencional, lo cual se diferencia de la simple desconexión o distracción, dicho cambio proviene del carácter de los objetos que intervienen, de esta forma siempre es más difícil cambiar la atención de un objeto a otro cuando la actividad precedente es más interesante que la actividad posterior.

Esta capacidad para oscilar o desplazar la atención puede ser considerado como un tipo de flexibilidad que se manifiesta en situaciones diversas, especialmente en las que tenemos que reorientar nuestra atención de forma apropiada porque nos hemos distraído o porque tenemos que atender a varios estímulos a la vez (García, 1997; Rubenstein, 1982; Orjales, 1999; mencionados en Ortiz,1996, p.58).

Si tomamos en cuenta estas descripciones sobre el proceso atentivo podemos afirmar que en los pacientes que podríamos llamarlos de etiología psicógena, lo que prioritariamente tendrían afectado sería el proceso de la atención y eso es lo que interfiere el recuerdo o evocación en su vida diaria, y que se confunde muchas veces como "olvidos ", así mejorando este trastorno de las esferas emocionales puede mejorar igualmente la atención selectiva, doble y simultánea inclusive, a la que podrían ser atribuidos estos "olvidos benignos", que son muy frecuentes en personas con fácil distraibilidad o muy cargadas de emociones negativas. En el Test de Recuerdo Selectivo de Buschke \& Altman, la categoría más sensible al proceso atentivo, es la categoría de Recuerdo a corto plazo, y por ende se justifica esta relación estadística encontrada. 
Otro de las descripciones que deseamos comentar es que la mayoría de los participantes en la muestra clínica es de género femenino lo cual no se aparta de la realidad de estudios anteriores, donde se observa que hay alta incidencia de trastornos de naturaleza afectiva-.emocional en mujeres. Tal como lo reportan otros estudios (Robustella. \& Acerbi, (2005) los problemas que ensombrecen la calidad de vida de la población femenina son: el dolor crónico relacionado con las enfermedades articulares y el malestar emocional.

Mayores cifras de trastornos afectivos y de ansiedad para las mujeres, está también presente en la epidemiología de la patología alimentaria.

Así también los registros encontrados en la realidad española (Montero et. al, 2004), donde se corrobora una prevalencia mayor por el género -vale decir- la presencia significativamente más elevada de cuadros depresivos, ansiosos y fóbicos entre las mujeres; mientras que entre los hombres son más frecuentes los trastornos de personalidad y los derivados del consumo de alcohol y otras sustancias, que no quedan bien recogidos en muchos de los instrumentos de detección.

En este caso de trastorno mixto ansiedad-depresión, materia de nuestro reporte, un $57.6 \%$ de la muestra estuvo constituido por pacientes de sexo femenino.

Otro de los hallazgos interesantes es que si se encontraron diferencias significativas entre el desempeño mnésico de la muestra de pacientes con el grupo no clínico, a través del Test de Recuerdo Selectivo, en todas las categorías de recuerdo ,a favor de la muestra del segundo grupo, lo que corrobora estudios anteriores (Llaja , 1983, 2000) que el Test de Recuerdo selectivo sigue siendo una medida válida y confiable en diferentes grupos muestrales, donde se hace necesario determinar si el desempeño del registro, almacenamiento, consolidación y evocación de los datos en el sistema de memoria está dentro de estándares normales, o si se trata de un problema de alguna de las fases de este proceso tan importante en el aprendizaje de cualquier edad o etapa de la vida. Además que es el instrumento que ha demostrado más eficacia en el diagnóstico neuropsicológico y diferencial de diversas entidades clínicas tanto en nuestra realidad como en otras a nivel internacional.

\section{CONCLUSIONES}

1. Los pacientes con trastorno mixto ansiedad-depresión no muestran déficit en el Sistema de memoria, comparados con una muestra homogénea no clínica.

2. Los pacientes con trastorno mixto ansiedad -depresión no muestran déficit en el registro, almacenamiento, consolidación ni evocación de los datos a largo plazo. 
3. Los pacientes de nuestra muestra de estudio sólo presentan déficit en la memoria a corto plazo, lo cual puede ser atribuido a un trastorno primario en el proceso de atención selectiva, (el trastorno mixto F41.2 interfiere en esta categoría de recuerdo a corto plazo)

4. Finalmente podemos afirmar que la evaluación neuropsicológica integral del sistema de Memoria a través del Test de Recuerdo Selectivo sigue siendo una medida diagnóstica válida y confiable de los desordenes del Sistema de Memoria asociados a trastornos de naturaleza psicógena.

\section{RECOMENDACIONES}

Como se sigue acentuando la incidencia de trastornos afectivo-emotivos, en nuestro medio, sobre todo en muestras poblacionales de género femenino, se sugiere elaborar programas de prevención y promoción de la salud: como cartillas terapéuticas del manejo del estrés (flagelo de este siglo)para ser aplicados tanto a nivel de Lima metropolitana, zonas urbano marginales como también rurales en provincias, para lo cual podría emplearse los medios de comunicación masiva: gráficos y de locución radial ; a nivel clínico- educativo (Escuela de Padres) y ayudarían significativamente los medios de tecnología e informática, incluso para que la información llegue a las grandes mayorías a través de redes sociales tan difundidas actualmente en distintos estratos sociales.

\section{REFERENCIAS}

Arbieto,K. (2008). Psicopedagogía.com. Recuperado el 19 de Noviembre del 2011 de: $\mathrm{http} / / \mathrm{www}$.psicopedagogia.com/atención

Díaz, M. (2009)Curso Taller Avanzado. Técnicas y métodos de Investigación Científica. Lima:Manual UPCH.

García,P. Llaja,V., Sarria,C, \& col.(2009) Aptitudes cognitivas y estrategias de memorización secuencial de los alumnos de un Centro Educativo privado de Comas. Lima: Revista de Investigación en Psicología, 12(1) 45-59.

Godoy,J.(1999). Psicología de la Salud: delimitación conceptual. En Simón, M. (Ed.). Manual de psicología de la Salud. Madrid: Biblioteca Nueva.

Hernandez, Fernandez y Baptista (2010). Metodología de la Investigación . Mexico: Mc Graw Hill.

Luria, A. (1980). Neuropsicología de la Memoria. Madrid: Blume.

Luria, A. (1974). El Cerebro en Acción. Barcelona: Fontanella.

Llaja, V.(1983). Amnesia Global Transitoria un Estudio Neruropsicologico . (Tesis Licenciatura). Universidad San Martín de Porres, Lima, Perú.

Llaja, V.(2000) Los estímulos familaires y la memoria verbal en niños de 5 años (Tesis Maestría). Universidad Inca Garcilazo de la Vega ,Lima, Perú. 
Llaja,V. Sarria,C.García ,P. (2009) Evaluación neuropsicológica del delirio y la incidencia de los factores de riesgo en pacientes de Cuidados Intensivos del HNERM. Lima: Revista de la Academia Peruana de Neurociencias del Perú. en prensa.Año 1. (2 ) 32-36

Llaja, V, Sarria,C, García, P. (2008) Déficit cognitivos en la enfermedad de Parkinson interpretados bajo el modelo informacional de la Personalidad. Lima: Revista de la Academia Peruana de Neurociencias del Perú. 1(1) 25-28.

Montero,I.\& col. (2004)Género y Salud Mental en un mundo cambiante. En: Gaceta Sanitaria (18), Supl.1 Barcelona: Scielo

Ortiz, P. (1998) El Nivel Consciente de la Memoria. Lima: Fondo Editorial de la Universidad de Lima.

Ortiz, P. (2004). Cuadernos de Psicobiología Social 1 Introducción a la Psicobiología del hombre . Lima: UNMSM..

Ortiz, P. (2004) Cuadernos de Psicobiología Social 6 El nivel Consciente de la Actividad Personal . Lima: UNMSM.

Ortiz, P. (1994) El Sistema de la Personalidad . Lima: Talleres Gráficos. UNMSM.

Ortiz ,P. (1996) La formación de la Personalidad. Lima: Dimaso Editores.

Ortiz,P. (2008). Educación y formación de la Personalidad. Lima: Fondo Editorial de la Universidad de Ciencias y Humanidades.

Reynolds, C. R. \& Hickman, J. A. (2004) Draw-A-Person Intellectual Ability Test for Children, Adolescents, and Adults (DAP:IQ). Austin: PRO-ED. Lindgren, S. D., \& Richman, L. D. (1984) Inmediate memory functions verbally deficient readingdisabled children. Journal of Learning Disabilities, 17, 222-225.

Richman, L. C.\& Lindgren, S.D. (1980) Patterns of intellectual ability in children with verbal deficits. Journal of Abnormal Child Psichology, 8, 65-75.

Robustella,V. \& Acerbi,E.(2005) Desordenes afectivos en la mujer. Revista Argentina de Clínica Neuropsiquiatría. XV(12), 391-401.

Rourke, B. P. (Ed.) (1985) Neuropsychology of learning disabilities. New York: Guilford Press.

Sarria, C, García, P., y Llaja, V.(2008) Compendio de Neuropsicología clínica .Pesquisas Neuropsicológicas . Lima: CEPREDIM.UNMSM

Sarria,C.,García,P. y Llaja, V. (2008).Test de Percepción Visomotor de Benton Revisado. Lima: CEPREDIM. UNMSM. 
